\title{
The Cultivation of the Rumen Ciliates Epidinium ecaudatum caudatum and Polyplastron multivesiculatum in vitro
}

\author{
By G. S. COLEMAN, JUDITH I. DAVIES AND \\ MARGARET A. CASH \\ Department of Biochemistry, Agricultural Research Council, \\ Institute of Animal Physiology, Babraham, Cambridge \\ (Received I3 June 1972; revised 31 July 1972)
}

\begin{abstract}
SUMMARY
Populations of over 400 Epidinium ecaudatum caudatum $/ \mathrm{ml}$ were maintained for over 18 months in the presence of bacteria, in a potassium phosphate-rich medium under $95 \% \mathrm{~N}_{2}+5 \% \mathrm{CO}_{2}$ with the daily addition of wholemeal flour and dried grass. Polyplastron multivesiculatum was also maintained at a population density of 45 to $70 / \mathrm{ml}$ under the same conditions, except that it was necessary to add each day a culture of another protozoon such as Epidinium ecaudatum caudatum, $E$. ecaudatum tricaudatum or Eudiplodinium maggii to give a population density of approximately $100 / \mathrm{ml}$. The epidinia at least were engulfed by the polyplastron up to Io being taken up each day; the minimum daily requirement for growth was one epidinium/polyplastron. Polyplastron multivesiculatum also grew in the absence of other protozoa on a sodium chloride-rich medium, and if these protozoa were inoculated into the potassium phosphate-rich medium in the presence of epidinia, there was a lag of 3 to 5 days before they began to engulf the epidinia. The effect of other variations in the culture conditions is also described.
\end{abstract}

\section{INTRODUCTION}

Although entodinia from the rumen have been successfully cultivated for long periods by several workers including Hungate (1943), Coleman (I960), Tompkin, Purser \& Weiser (I966), Jarvis \& Hungate (I968) and Coleman (I969), there have been fewer investigations on the growth of the larger rumen entodiniomorphid protozoa. Hungate (I942, 1943) maintained several diplodinia, including Diplodinium (Polyplastron?) multivesiculatum, for over a year at low population densities. Gutierrez \& Davies (1962) cultivated Epidinium ecaudatum in vitro for 5 months, but obtained populations of only $\mathrm{I} 200 / \mathrm{ml}$ and did not investigate the effect of different cultural conditions on this density. Clarke (1963) maintained Eremoplastron bovis in unspecified numbers for 5 months and mixed entodiniomorphs for somewhat longer, while Mah (I964) maintained up to I 000 Ophryoscolex purkynei/ml for nearly 3 years, with a mean generation time of $24 \mathrm{~h}$.

The present paper describes the cultivation of Epidinium ecaudatum caudatum and Polyplastron multivesiculatum in the presence of bacteria in buffered salts medium with wholemeal flour and dried grass as the sources of carbohydrate. Under certain conditions $P$. multivesiculatum had an absolute requirement for the epidinia for growth and died rapidly without them.

Preliminary communications gave the growth requirements of these ciliates immediately after their isolation from the rumen (Coleman, I970, 1971). 


\section{METHODS}

Basalmineral salt solutions. 'Caudatum-type' salt solution contained (g/I00 ml): $\mathrm{K}_{2} \mathrm{HPO}_{4}$, $0.63 ; \mathrm{KH}_{2} \mathrm{PO}_{4}, 0.50 ; \mathrm{NaCl}, 0.065 ; \mathrm{CaCl}_{2}$ (dried), $0.0045 ; \mathrm{MgSO}_{4} .7 \mathrm{H}_{2} \mathrm{O}, 0.009 ; \mathrm{CH}_{3} \mathrm{COONa}_{\text {, }}$ 0.075. 'Simplex-type' salt solution contained (g/I00 ml): $\mathrm{K}_{2} \mathrm{HPO}_{4}, 0.49 ; \mathrm{KH}_{2} \mathrm{PO}_{4}, 0.38$; $\mathrm{NaCl}, 0.049 ; \mathrm{CaCl}_{2}$ (dried), $0.0035 ; \mathrm{MgSO}_{4} .7 \mathrm{H}_{2} \mathrm{O}, 0.007 ; \mathrm{NaHCO}_{3}, 0.66$. 'Dilute simplextype' salt solution contained (g/IOO ml): $\mathrm{K}_{2} \mathrm{HPO}_{4}, 0.3 \mathrm{I} ; \mathrm{KH}_{2} \mathrm{PO}_{4}, 0.24 ; \mathrm{NaCl}, 0.03 \mathrm{I}$; $\mathrm{CaCl}_{2}$, (dried), $0.0022 ; \mathrm{MgSO}_{4} \cdot 7 \mathrm{H}_{2} \mathrm{O}, 0.0044 ; \mathrm{NaHCO}_{3}, 0.66$. 'Hungate-type' salt solution, based on that of Hungate (I942), contained (g/IOO ml): $\mathrm{NaCl}, 0.5 ; \mathrm{CH}_{3} \mathrm{COONa}, 0.15$; $\mathrm{K}_{2} \mathrm{HPO}_{4}, 0 . \mathrm{I} ; \mathrm{KH}_{2} \mathrm{PO}_{4}, 0.03$.

Culture media. All experiments were carried out in 50 or $100 \mathrm{ml}$ centrifuge tubes approximately three-quarters filled with salts medium to which was added $\mathrm{L}$-cysteine hydrochloride (neutralized immediately before use; final concentration $0.02 \%, \mathrm{w} / \mathrm{v}$ ) and sometimes $10 \%$ fresh or autoclaved rumen fluid. Ninety-five per cent $(\mathrm{v} / \mathrm{v}) \mathrm{N}_{2}+5 \%(\mathrm{v} / \mathrm{v}) \mathrm{CO}_{2}$ (for caudatum or Hungate-type salts), or $\mathrm{CO}_{2}$ (for simplex-type or dilute simplex-type salts) was then bubbled vigorously through the medium for 2 to $3 \mathrm{~min}$ and the tubes stoppered immediately with a rubber bung.

Rumen fluid fractions. Fresh rumen fluid from which the protozoa had been removed was prepared as described by Coleman (I958). Autoclaved rumen fluid (ARF) was prepared as described by Coleman (1969).

Initial isolation of protozoa. This was carried out by picking out single protozoa from crude rumen fluid as described by Coleman (1971).

Culture of Epidinium ecaudatum caudatum. Coleman (197I) found that in newly established cultures epidinia grew best on dilute simplex-salts under $100 \% \mathrm{CO}_{2}$, but older cultures grew better in media equilibrated with gas containing $5 \% \mathrm{CO}_{2}$. The standard cultural conditions used for over a year to grow epidinia from the second isolation (see below) are as follows. The protozoa were maintained in $100 \mathrm{ml}$ centrifuge tubes in caudatum salts with $0.02 \%$ L-cysteine, but no rumen fluid, under $95 \% \mathrm{~N}_{2}+5 \% \mathrm{CO}_{2}$, at $39{ }^{\circ} \mathrm{C}$. Wholemeal flour $(0.2 \mathrm{ml}$ of $\mathrm{I} .5 \%, \mathrm{w} / \mathrm{v}$, aqueous suspension) and ground, dried grass (about $2 \mathrm{mg}$ ) were added each day. Twice a week the culture was mixed, half poured into a clean tube and the liquid in each tube made up to the original volume with fresh medium.

Culture of Polyplastron multivesiculatum. This protozoon was maintained routinely both in the presence and absence of epidinia. In the presence of epidinia Polyplastron multivesiculatum was grown in $50 \mathrm{ml}$ centrifuge tubes in caudatum-type salts with $0.02 \% \mathrm{~L}$ cysteine, but no rumen fluid, under $95 \% \mathrm{~N}_{2}+5 \% \mathrm{CO}_{2}$, at $39{ }^{\circ} \mathrm{C}$. Wholemeal flour (O.I ml of $\mathrm{I} \cdot 5 \%, \mathrm{w} / \mathrm{v}$, aqueous suspension), ground, dried grass (about $\mathrm{I} \mathrm{mg}$ ) and $\mathrm{I} \mathrm{ml}$ of a culture of Epidinium ecaudatum caudatum (or other protozoa) were added each day. In addition, twice a week the cultures were diluted with an equal volume of fresh medium as above.

In the absence of epidinia Polyplastron multivesiculatum was grown in Hungate-type salts, without cysteine or rumen fluid, in $100 \mathrm{ml}$ centrifuge tubes at $39^{\circ} \mathrm{C}$. Wholemeal flour (I drop $\mathrm{I} \cdot 5 \%, \mathrm{w} / \mathrm{v}$, aqueous suspension) and ground, dried grass $(2 \mathrm{mg})$ were added each day. In addition, twice a week the cultures were diluted with an equal volume of fresh medium as above.

Protozoal counts. The number of epidinia in a culture was estimated by blowing from a pipette $\mathrm{I} \cdot 0 \mathrm{ml}$ of the culture into $\mathrm{I} \cdot 0 \mathrm{ml} 0.02 \mathrm{M}$-iodine and counting microscopically all the protozoa in $0.1 \mathrm{ml}$ of the mixture. For Polyplastron multivesiculatum cultures, where the numbers were frequently less than $100 / \mathrm{ml}$, a suitable volume of culture was mixed with an equal volume of $0.02 \mathrm{M}$-iodine in a $4 \times \frac{1}{2}$ inch tube and all the protozoa present counted 
under an inverted microscope (Olympus Optical Co. Ltd, Tokyo, Japan-model CK). Only those protozoa which showed no signs of disintegration were counted. To judge the effect of a change in growth medium at least 4 weeks were allowed to elapse before the number of protozoa was estimated. Unless otherwise stated, the number of protozoa was always estimated immediately before the dilution of that culture.

\section{RESULTS}

\section{Cultivation of Epidinium ecaudatum caudatum}

For the continued maintenance of Epidinium ecaudatum caudatum in the presence of bacteria it was necessary to add fresh wholemeal flour to the cultures each day. When no such addition was made all the protozoa died within $48 \mathrm{~h}$. It was not necessary for the maintenance of the protozoa to dilute the cultures frequently with fresh medium. Cultures remained alive for over 4 weeks without the addition of fresh medium provided that wholemeal flour was added each day.

Epidinium ecaudatum caudatum was isolated originally in simplex-type salts medium containing prepared, fresh, rumen fluid $\left(10 \%\right.$, v/v) under $100 \% \mathrm{CO}_{2}$, because single protozoa picked directly from crude rumen contents grew better in this medium than in caudatumtype salts medium containing 10 $\%(\mathrm{v} / \mathrm{v})$ prepared, fresh, rumen fluid under $95 \% \mathrm{~N}_{2}+5 \%$ $\mathrm{CO}_{2}$. However, after 3 months with twice-weekly dilution of the cultures, the population density in the former medium declined slowly from about $1000 / \mathrm{ml}$, and after a further year all the protozoa were dead. Investigations to find the optimal growth conditions carried out I to 4 months after the initiation of the culture showed that, at this time, caudatum-type salts medium containing no rumen fluid supported the highest population density and so some stock cultures were transferred to this medium. Despite sporadic improvements in the number of protozoa present, the population density supported by this medium also declined until, regardless of the cultural conditions, all the protozoa from the isolate died about the same time. Throughout the last few months of the life of this isolate, attempts to stimulate growth by variations in the salt concentration ( 40 to $150 \%$ of standard), $\mathrm{CO}_{2}$ tension $\left(5 \%\right.$ or $\left.100 \% \mathrm{CO}_{2}\right)$, type of starch (rice or maize starch or wholemeal flour), presence or absence of $10 \%(\mathrm{v} / \mathrm{v})$ autoclaved or prepared, fresh, rumen fluid and in the frequency with which cultures were diluted with fresh medium (twice a week or once a fortnight) were all unsuccessful. The death of this isolate may have been caused in part by an increased susceptibility of the epidinia to the presence of excess starch in the bottom of the tube. If the starch added to the medium during the daily feeding routine was not engulfed immediately by the protozoa, it remained in the bottom of the tube next to the protozoa and was fermented by the bacteria present. Normally protozoa were not harmed by the fermentation products provided that only a small amount of starch was present, but it was difficult to judge the correct amount of wholemeal flour to add each day. If too much was added all the protozoa were frequently dead I6 h later, whereas if too little was added they died of starvation. A second isolate, which was made after the death of the first, did not decline in the same way and I 8 months after it was initiated 400 protozoa/ml were present under standard conditions and this population density remained constant for over 6 months.

Clone cultures of Epidinium ecaudatum tricaudatum and the cattle form of E. ecaudatum caudatum were isolated from a Dexter cow and at the time of writing have been maintained for 3 months on simplex-type salts medium, containing $10 \%(\mathrm{v} / \mathrm{v})$ prepared, fresh, rumen fluid, at population densities of $1000 / \mathrm{ml}$ and $500 / \mathrm{ml}$ respectively. 


\section{Morphology}

Epidinium ecaudatum caudatum on which all the cultural studies have been carried out was taken from a rumen population (Type B, Eadie, I962) present in a Clun Forest sheep fed on hay and oats, and measured I09 $\pm 9 \mu \mathrm{m} \times 47 \pm 3 \mu \mathrm{m}(n=10$; range 98 to $\mathrm{I} 20 \mu \mathrm{m} \times 4 \mathrm{I}$ to $52 \mu \mathrm{m}$ ) when removed from the sheep. The protozoon's caudal spine gradually became smaller during the first few months in culture and disappeared after 4 to 5 months. After 12 months in culture protozoa from the second isolate, grown under standard conditions, measured I I $8 \pm \mathrm{I} 4 \mu \mathrm{m} \times 62 \pm 5 \mu \mathrm{m}$ ( $n=10$; range 104 to $148 \mu \mathrm{m} \times 55$ to $72 \mu \mathrm{m}$ ), showing that in culture the epidinia were less uniform in size. On initial isolation this protozoon had a structure similar to that described for this species by Sharp (1914), except that the single caudal spine, which was curved in the living organism, was shorter, i.e. it was $20 \cdot 0 \pm 5.6 \%$ of the length of the remainder of the protozoon compared with 33 to $50 \%$. The short spination is characteristic of epidinia present in sheep. Of the two cattle species one was identical with $E$. caudatum tricaudatum (Sharp, 1914) but the other, which had a long straight caudal spine $(42 \pm 8 \%$ of the length of the remainder of the protozoon), did not resemble any of the pictures of epidinia published by Crawley (I923), Sharp (I9I4), Dogiel (I927), or Kofoid \& MacLennan (I933), because they were all taken from fixed material in which the spine always appears to be curved regardless of its natural configuration. In the living protozoon the spine extends straight backwards from the posterior end of the organism. The spine was still present with the same configuration after 3 months in culture, although Clarke (1963) found that mixed epidinia grown in vitro lost these spines after 7 months in culture. This protozoon is referred to hereafter as the 'cattle form' of E. ecaudatum caudatum. The width of the spine at its base was $6.6 \pm 0.7 \mu \mathrm{m}$ in the sheep form and $8.7 \pm \mathrm{I} \mu \mathrm{m}$ in the cattle form.

\section{Effect of medium constituents}

The effects of varying each constituent of the standard medium (see Methods) and of adding other materials are shown below. The actual experiments were carried out with the first isolate of the sheep form but the principal conclusions were confirmed with the second isolate.

Wholemeal flour. The daily addition of wholemeal flour to a final concentration of 0.03 , 0.07 or $0.10 \mathrm{mg} / \mathrm{ml}$ resulted in protozoal populations of 420,560 and 800 epidinia/ml respectively. Wholemeal flour was not replaceable by an equal quantity of a purified starch, such as rice starch, for the long-term maintenance of the protozoa, although some epidinia remained alive for up to 20 days in the presence of rice starch as sole source of carbohydrate. Epidinium ecaudatum caudatum therefore resembles Entodinium simplex (Coleman, 1969) rather than Entodinium caudatum (Coleman, 1960) which grew well on rice starch.

Rumen fluid. Although the initial isolation was carried out in the presence of $10 \%$ $(\mathrm{w} / \mathrm{v})$ prepared, fresh, rumen fluid this was not essential for the growth of established cultures and population densities obtained with 0 , 10 \% prepared, fresh, rumen fluid or $10 \%$ autoclaved rumen fluid and the daily addition of $0.07 \mathrm{mg} / \mathrm{ml}$ wholemeal flour were 560,400 and 460 ciliates $/ \mathrm{ml}$. The population densities in the presence of the rumen fluids could be raised to that in their absence by doubling the amount of wholemeal flour added each day. As a result of these observations stock cultures were maintained thereafter without rumen fluid. Epidinium ecaudatum caudatum therefore differs from both the entodinia studied previously (Coleman, 1960, 1969) where the presence of autoclaved rumen fluid increased the population density almost ten times. 


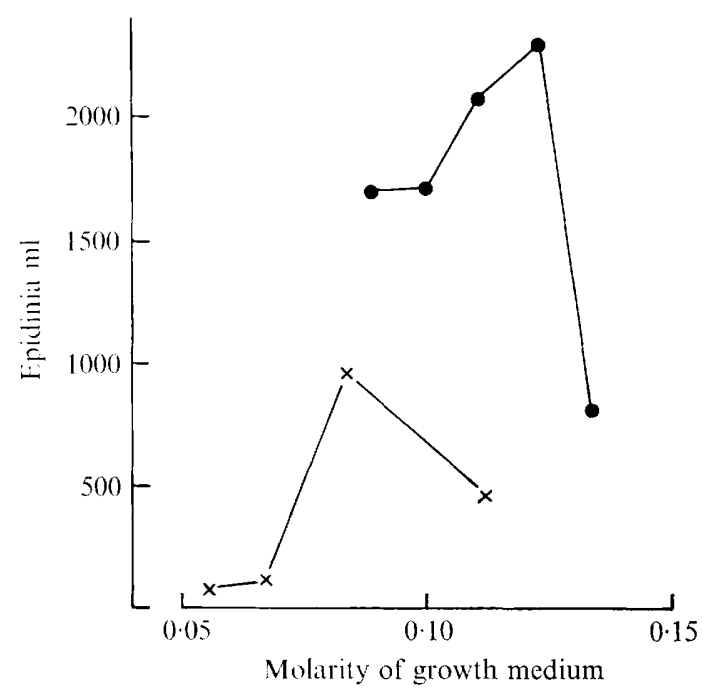

Fig. I. The effect of salt concentration on the growth of Epidinium ecaudatum caudatum from a small inoculum in the presence of $10 \%$ prepared, fresh, rumen fluid. The protozoa were grown on caudatum-type salts medium under $95 \% \mathrm{~N}_{2}+5 \% \mathrm{CO}_{2}(\times-\times)$ or on simplex-type salts medium under $100 \% \mathrm{CO}_{2}(-)$, the salt concentration being varied by replacement of the salts solution by water or double strength salts except that the concentration of $\mathrm{NaHCO}_{3}$ remained unchanged to maintain the $\mathrm{pH}$ constant. The protozoa for inoculation were grown on caudatum-type salts medium and added at a population density of $100 / \mathrm{ml}$. The cultures were fed with wholemeal flour and dried grass each day and the number of epidinia estimated after 7 days.

Dried grass. This was stimulatory but not essential for the growth of epidinia, because after 6 months of growth in its absence the population density was $180 / \mathrm{ml}$ compared with $560 / \mathrm{ml}$ in its presence.

Temperature. Epidinium ecaudatum caudatum was even more sensitive to cooling than the entodinia and was killed by exposure for $18 \mathrm{~h}$ to a temperature of $30^{\circ} \mathrm{C}$; the normal growth temperature was $39^{\circ} \mathrm{C}$.

Basal mineral salt solutions and $\mathrm{CO}_{2}$ tension. To determine the optimum salt concentration for growth, epidinia were grown from a small inoculum $(60 / \mathrm{ml})$ in simplex- or caudatumtype salts media, the concentrations of which were varied by replacement of some of the complete salts mixture by an equal volume of water or double strength salts medium. However with simplex-type salts medium the concentration of $\mathrm{NaHCO}_{3}(0.077 \mathrm{M})$, which had to be added to maintain the $\mathrm{pH}$ constant at $6 \cdot 7$ under a $\mathrm{CO}_{2}$ atmosphere, remained unchanged. Fig. I shows that, in an experiment which was carried out 3 months after the initiation of the first culture, the optimum concentration of caudatum salts for epidinia grown under $95 \%$ $\mathrm{N}_{2}+5 \% \mathrm{CO}_{2}$ was $0.08 \mathrm{M}$ (calculated from the composition of these salts), whereas for simplex salts under $100 \% \mathrm{CO}_{2}$ it was $0.12 \mathrm{M}$. Hungate (I955) found a similar effect of partial pressure of carbon dioxide on the optimum salt concentration for the growth of diplodinia. So, dilute rather than normal simplex-type salts medium was used thereafter for the growth of epidinia under $100 \% \mathrm{CO}_{2}$, but this change did not prevent the eventual death of the first isolate. Although the results shown in Fig. I suggest that growth on caudatum-type salts medium was much poorer than that on simplex-type salts medium, there was no significant difference in the number of protozoa (i.e. about $400 / \mathrm{ml}$ ) present when they were maintained for over a year in these two media under standard conditions with twice-weekly dilution of the culture. 


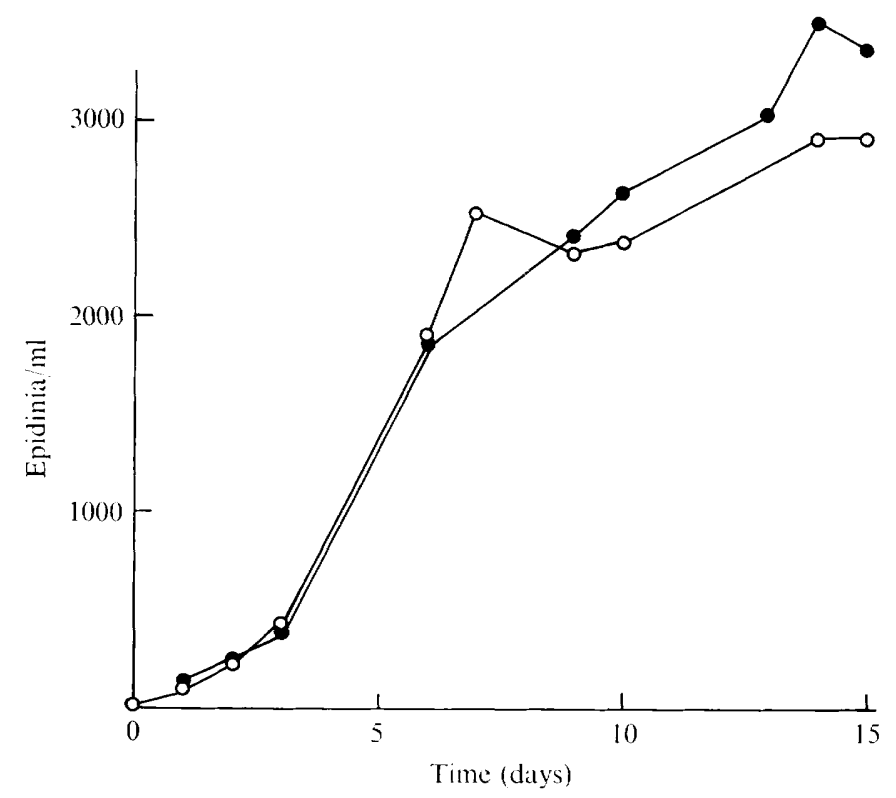

Fig. 2. The effect of autoclaved rumen fluid on the growth of Epidinium ecaudatum caudatum from a small inoculum. The experiment was carried out in $50 \mathrm{ml}$ centrifuge tubes containing dilute simplextype salts medium inoculated with $\mathrm{I} \mathrm{ml}$ of a standard culture and to which $0 . \mathrm{I} \mathrm{ml} \mathrm{I} \cdot 5 \%$ wholemeal flour suspension and I mg dried grass were added each day. - N , No rumen fluid; $\mathrm{O}-\mathrm{O}$, $+10 \%$ autoclaved rumen fluid.

\section{Growth from a small inoculum}

As the protozoa were normally maintained by twice weekly dilution of the culture with an equal volume of fresh medium, it was of interest to measure protozoal growth when a small inoculum $(3 \%)$ was added to fresh medium. For this experiment $\mathrm{I} \mathrm{ml}$ of a standard culture in dilute simplex-type salts medium under $100 \% \mathrm{CO}_{2}$ was inoculated into $30 \mathrm{ml}$ of the same medium in a $50 \mathrm{ml}$ centrifuge tube and the number of protozoa counted in I $\mathrm{ml}$ samples removed daily when the cultures were fed. There was no obvious lag before protozoal growth began and over the first six days the mean generation time was I $\cdot$ I days (Fig. 2); the presence of autoclaved rumen fluid had no effect on this rate. This mean generation time compares favourably with times of $\mathrm{I} \cdot 8$ and I 6 days for Entodinium caudatum and E. simplex respectively (Coleman, 1960, 1969).

\section{Cultivation of Polyplastron multivesiculatum}

Polyplastron multivesiculatum grew well in simplex-type medium in the presence of epidinia provided that wholemeal flour and dried grass were added to the medium each day. It was relatively easy to obtain growth of a single protozoon (picked from crude rumen fluid with a micromanipulator) added to any culture of Epidinium ecaudatum caudatum. In preliminary experiments (Coleman, I97r) the epidinia were grown on simplex-type medium under $100 \% \mathrm{CO}_{2}$ but when it had been found that $P$. multivesiculatum grew better on caudatum-type salts under $95 \% \mathrm{~N}_{2}+5 \% \mathrm{CO}_{2}$, the epidinia used for the initial isolation were grown under these conditions. Although it was of interest to study the metabolism of $P$. multivesiculatum predatory upon epidinia, it was also important to study this protozoon growing in the absence of other protozoa to determine if its metabolic capabilities were the same when grown under the two different conditions. It was not possible to grow $P$. multivesiculatum in the absence of epidinia just by omitting the latter from the medium because 
the former always died, except on the one occasion reported previously (Coleman, 197I). It was very difficult to obtain growth of single $P$. multivesiculatum organisms in any of the media used for entodinia or epidinia (Coleman, I97I) in the presence or absence of rumen fluid, except in Hungate-type salts medium which had been inoculated the previous day with bacteria from crude rumen fluid or an established protozoal culture. Under these conditions approximately 10 \% of single protozoal isolates grew and divided. The subsequent maintenance of the cultures was as described under 'Methods'. At all stages it was very important not to add too much wholemeal flour to the cultures. However, even with this precaution the protozoa occasionally all died inexplicably, and no culture was ever maintained for more than a year in the absence of epidinia.

\section{The size of the organism}

The protozoa were taken from the rumen of a Clun Forest sheep fed on hay and oats, with a type A population (Eadie, 1962) and measured $175 \pm 19 \mu \mathrm{m} \times 127 \pm 8 \mu \mathrm{m}(n=10)$ when removed from the sheep. The protozoa grown in the presence of the epidinia were always densely filled and measured $205 \pm \mathrm{I} 3 \mu \mathrm{m} \times \mathrm{I} 23 \pm \mathrm{I} 4 \mu \mathrm{m}(n=\mathrm{I} 0)$ after $\mathrm{I} 2$ months in culture. Polyplastron multivesiculatum grown on Hungate-type salts medium in the absence of epidinia was smaller and measured only $123 \pm 9 \mu \mathrm{m} \times 98 \pm 9 \mu \mathrm{m}(n=10)$. Eadie (1967) also observed that when $P$. multivesiculatum was introduced into a rumen containing protozoa upon which it was predatory (a type B population) it increased in size and became filled with dense material.

\section{Effect of medium constituents on growth in presence of epidinia}

The effects of varying each constituent of the standard medium (see 'Methods') are shown below.

Basal mineral salts and $\mathrm{CO}_{2}$ tension. Polyplastron multivesiculatum was isolated initially on simplex-type salts medium under $100 \% \mathrm{CO}_{2}$, but after 2 months, growth became progressively poorer until 4 months later it was no longer worthwhile to continue with this medium. However, if at any stage the medium was changed to caudatum-type salts medium under $95 \% \mathrm{~N}_{2}+5 \% \mathrm{CO}_{2}$ the population density increased and at the time of writing $P$. multivesiculatum has been maintained for over a year under these conditions.

Rumen fluid. The addition of rumen fluid to caudatum-type salts medium under $95 \% \mathrm{~N}_{2}+$ $5 \% \mathrm{CO}_{2}$ did not stimulate protozoal growth and population densities of 63,49 and 47 protozoa $/ \mathrm{ml}$ were found without rumen fluid, or with $10 \%$ autoclaved or $10 \%$ prepared, fresh, rumen fluid respectively. Polyplastron multivesiculatum was therefore grown without rumen fluid.

Wholemeal flour. The daily addition of wholemeal flour to a final concentration of $\mathrm{o}$, $0.03,0.06,0.1$ or $0.25 \mathrm{mg} / \mathrm{ml}$ resulted in population densities of $0,4 \mathrm{I}, 48,45$ and 40 ciliates/ $\mathrm{ml}$ respectively. This similarity in population densities obtained in the presence of all concentrations of wholemeal flour tested suggested that the requirement of these protozoa for wholemeal flour was met by the lowest concentration and that at the higher concentrations something else was limiting growth. The wholemeal flour was not completely replaceable by rice starch, the protozoa dying after 25 days compared with 6 days in the absence of any added starch. This means that the polysaccharide present in the epidinia added each day would not support even a limited protozoal population.

Dried grass. Omission of dried grass from the daily feeding resulted in death of the culture in 10 days provided that the epidinia added each day were grown in the absence of grass.

Epidinia. The daily addition of o, 30, 75 or 150 epidinia/ml (added as a culture of Epi- 

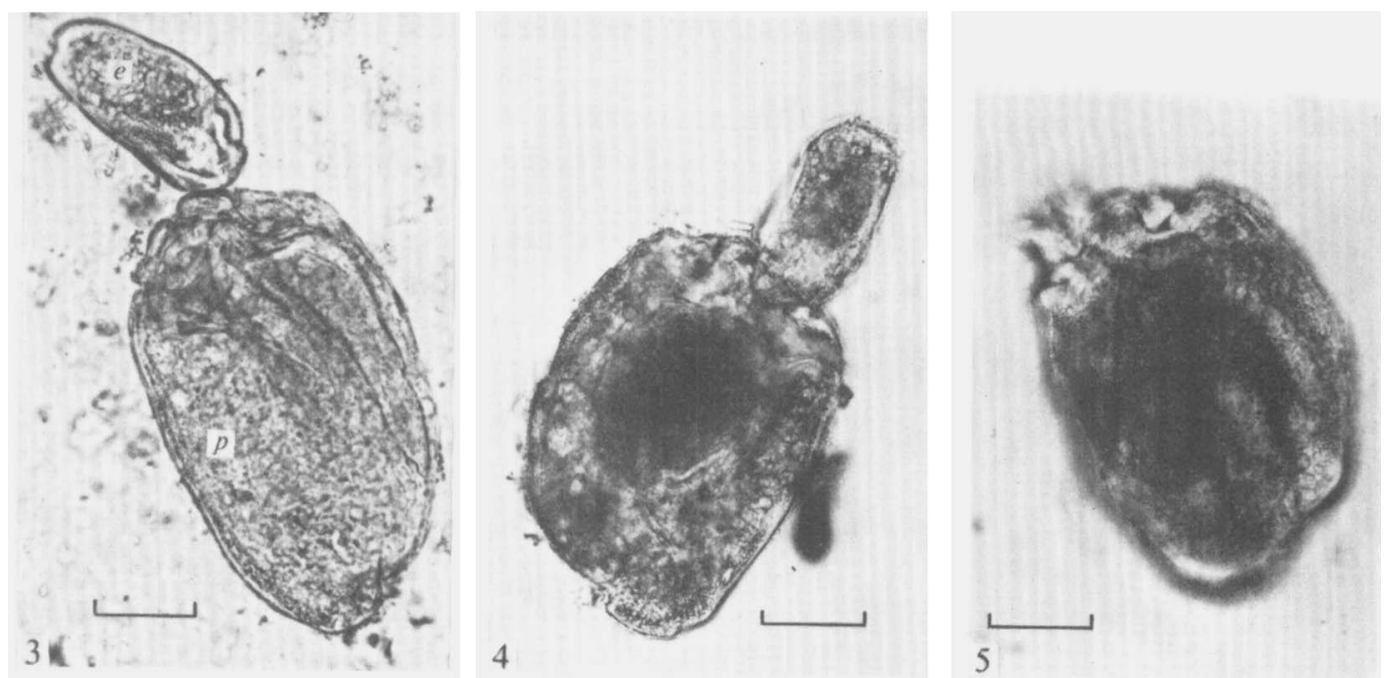

Fig. 3. A photomicrograph of Polyplastron multivesiculatum ( $p$ ) about to engulf an Epidinium ecaudatum caudatum (e). Epidinia were usually taken in anterior end first but the reverse was occasionally seen.

Fig. 4. An epidinium in the process of being engulfed.

Fig. 5. An epidinium inside a polyplastron. Bar markers $=200 \mu \mathrm{m}$.

dinium ecaudatum caudatum) to a culture resulted in Polyplastron multivesiculatum populations of o, Io, 30 and $48 / \mathrm{ml}$ respectively. However, the ratio of epidinia added daily to $P$. multivesiculatum organisms present, which was approximately 3 in the above experiment, varied from 0.5 to 4 in different experiments under the same conditions. In each case all the epidinia had disappeared from the medium within $24 \mathrm{~h}$ of their addition. Except for the one experiment mentioned above, cultures to which epidinia were not added each day died within a few days (Fig. 6), although a few polyplastrons occasionally survived for 2 weeks. As Eadie (1967) showed that $P$. multivesiculatum was predatory upon protozoa in a type B population it was likely that the epidinia in the present experiments were being engulfed by the polyplastrons. Unfortunately, these protozoa were normally densely filled with storage material and it was not possible to observe epidinia inside them. If, however, the polyplastrons were starved of epidinia for 2 days they became transparent and then on the addition of epidinia to the culture individual polyplastrons could be seen after $30 \mathrm{~min}$ of incubation to contain one, or occasionally two, epidinia. Fig. 3 to 5 show three stages in the engulfment of an epidinium by a polyplastron. Further observation on the number of epidinia engulfed was not possible as the polyplastrons became densely filled again. These results show that at least some of the epidinia were killed by engulfment, but it is uncertain if this was the only reason for their disappearance.

The maximum number of epidinia that could be engulfed per day was determined by inoculation of 5 polyplastrons $/ \mathrm{ml}$ into medium containing 450 epidinia/ml initially. Fig. 6 shows that in 3 days (days 2 to 5 ) the number of polyplastrons increased from 2 to $42 / \mathrm{ml}$, while the number of epidinia decreased from 700 to $40 / \mathrm{ml}$. Over the last $24 \mathrm{~h}$ of this period an average of 33 polyplastrons $/ \mathrm{ml}$ engulfed or otherwise destroyed at least 380 epidinia $/ \mathrm{ml}$, assuming that none of the epidinia divided before they were engulfed. Each polyplastron was therefore capable of killing at least Io epidinia/day. Once all the epidinia had disappeared, the number of polyplastrons declined, although growth began again if further epidinia were added (Fig. 6). 


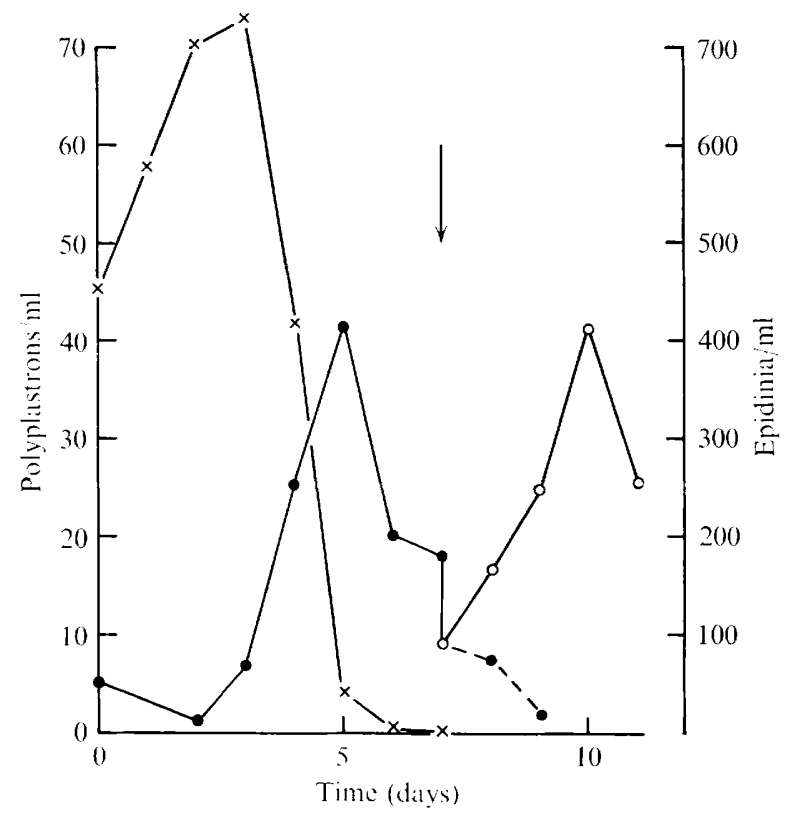

Fig. 6. The disappearance of Epidinium ecaudatum caudatum $(\times-\times)$ during the growth of Polyplastron multivesiculatum (grown previously in the presence of epidinia) (- - $)$ from a small inoculum in simplex-type salts medium containing $10 \%$ prepared, fresh, rumen fluid under $\mathrm{CO}_{2}$. At the arrow, when all the epidinia had disappeared, the culture was diluted with an equal volume of fresh medium and divided between two tubes to one of which was added 330 epidinia/ml. Number of polyplastrons: ---0 , without added epidinia; $\bigcirc-0$, with added epidinia.

The specificity of the requirement for epidinia varied with different isolates of Polyplastron multivesiculatum. The first isolate, which was grown from a single organism on the first strain of Epidinium ecaudatum caudatum, would not grow on Entodinium caudatum or E. simplex grown in vitro (Coleman, 1960, I969), on mixed entodinia from the rumen or on the second strain of epidinia, and therefore died when the first strain of epidinia died. Experiments carried out during the first few months of the life of this isolate of Polyplastron showed that epidinia broken by sonication supported $25 \%$ as many polyplastrons as intact epidinia. Epidinia killed by heating at $60^{\circ} \mathrm{C}$ for $5 \mathrm{~min}$, or by the addition of the minimum amount of iodine, supported $50 \%$ of this number for at least 2 weeks under standard conditions. The second isolate of Polyplastron multivesiculatum, which was isolated on the second strain of epidinia, would also not grow on the entodinia, but when first tested after a year in culture would grow in the presence of a clone culture of a species of Eudiplodinium (probably Eudiplodinium maggii). This protozoon, which was grown under the conditions used for Entodinium simplex (Coleman, 1969), was engulfed by the polyplastrons and 10 to $20 / \mathrm{ml}$ had to be added each day to maintain the polyplastrons. Under these conditions only large polyplastrons, which were the same size as those grown in the presence of epidinia, were found. However, polyplastrons could not be maintained indefinitely under these conditions and although population densities of up to $30 / \mathrm{ml}$ were obtained initially these declined after 2 months and after 4 months all the polyplastrons were dead. During this initial period each polyplastron engulfed, or otherwise destroyed, a maximum of nine eudiplodinia/day.

When it was first tested, after 15 months in culture, this second isolate of Polyplastron multivesiculatum also grew in the presence of a clone culture of a species of Diplodinium, 
which in culture resembled Diplodinium monocanthum. However, population densities of polyplastrons of less than $1 / \mathrm{ml}$ were obtained. The diplodinia never disappeared during the 3 months that the culture was maintained, and 200 to $400 / \mathrm{ml}$ were always present under standard growth conditions even though fresh diplodinia were not added each day. Although the average size of the polyplastrons under these conditions was $177 \pm 23 \mu \mathrm{m} \times 103 \pm 14 \mu \mathrm{m}$ $(n=10)$ individual protozoa varied in size from $\mathrm{I} 27 \times 88 \mu \mathrm{m}$ to $205 \times \mathrm{I} 24 \mu \mathrm{m}$, suggesting that only some individuals (the largest) were predatory upon the diplodinia.

The effect of clone cultures of Epidinium ecaudatum caudatum (cattle form) and $E$. ecaudatum tricaudatum on the growth of polyplastrons was also investigated. These epidinia, which only became available 18 months after the initial isolation of the polyplastrons and which were grown under the conditions used for Entodinium simplex (Coleman, 1969) were used to replace Epidinium ecaudatum caudatum (sheep form) under standard growth conditions. These new epidinia had to be added each day to the culture of Polyplastron multivesiculatum which was maintained at population densities similar to those obtained previously with $E$. ecaudatum caudatum (sheep form). Protozoa from a clone culture of Diploplastron affine (grown on caudatum-type salts medium, without rumen fluid, under $95 \% \mathrm{~N}_{2}+5 \% \mathrm{CO}_{2}$, with the daily addition of wholemeal flour and dried grass) did not support growth of polyplastrons and these died at the same rate as if no other protozoa had been added.

\section{Effect of medium constituents on growth in absence of epidinia}

Basal mineral salts and $\mathrm{CO}_{2}$ tension. As mentioned above, Polyplastron multivesiculatum only grew in the absence of other protozoa on Hungate-type salts medium under $95 \%$ $\mathrm{N}_{2}+5 \% \mathrm{CO}_{2}$. Attempts to grow $P$. multivesiculatum initially isolated on this medium in caudatum-type salts medium under $95 \% \mathrm{~N}_{2}+5 \% \mathrm{CO}_{2}$ or on simplex-type salts medium under $100 \% \mathrm{CO}_{2}$ in the presence or absence of rumen fluid were unsuccessful.

Rumen fluid. The addition of $10 \%$ prepared, fresh, rumen fluid or autoclaved rumen fluid did not increase the population density.

Wholemeal flour. As the population density was usually i to 5 protozoa $/ \mathrm{ml}$ the optimum amount of wholemeal flour added each day was about $0.05 \mathrm{mg} / \mathrm{ml}$. The protozoa were then actively motile in the bottom of the tube, but if more flour was added each day they became very sluggish and were liable to die unexpectedly. In one experiment a few protozoa survived for several months under standard conditions in the absence of wholemeal flour with just the daily addition of dried grass.

Dried grass. Omission of dried grass from the daily regimen resulted in death of the protozoa in 7 to Io days.

Epidinia. As Polyplastron multivesiculatum grew much better with Epidinium ecaudatum caudatum it was of interest to determine the effect of epidinia on the growth of $P$. multivesiculatum previously grown without them. A culture of $P$. multivesiculatum $(30 \mathrm{ml})$ grown in Hungate-type salts medium in the absence of epidinia was diluted with an equal volume of caudatum-type salts medium and $10 \mathrm{ml}$ placed in each of two $\mathrm{I} 50 \times 15 \mathrm{~mm}$ tubes: the diluted culture contained 2 ciliates $/ \mathrm{ml}$. To one of these tubes, and to a similar one containing the same medium, was added $\mathrm{I} \cdot 0 \mathrm{ml}$ of a culture of epidinia, to give a final population density of 42 epidinia/ml. The protozoa in each tube were then fed with $0 . \mathrm{I} \mathrm{ml} \mathrm{I} \cdot 5 \%$ wholemeal flour and I $\mathrm{mg}$ dried grass each day and all the $P$. multivesiculatum and E. ecaudatum caudatum present in the tube counted under an inverted microscope. Fig. 7 shows that in the presence of epidinia the number of $P$. multivesiculatum did not increase until the protozoa had been in the same culture for more than 3 days ( 5 days in one experiment) after which 


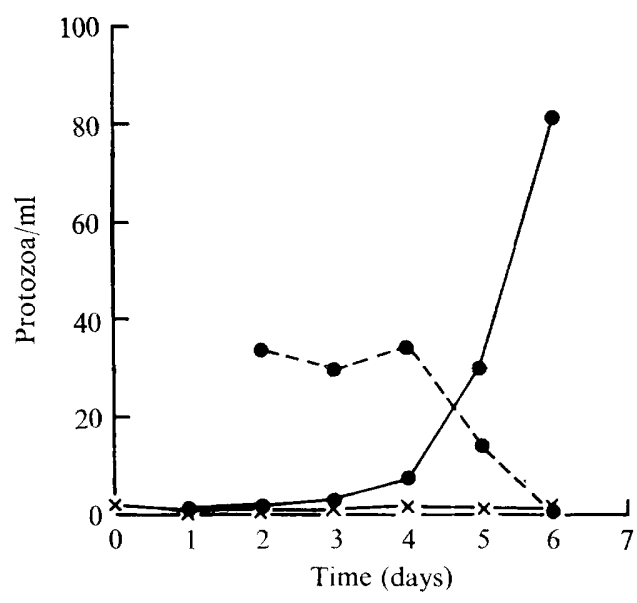

Fig. 7. Effect of the presence $(-\infty)$ ) or absence $(\times--\times)$ of Epidinium ecaudatum caudatum on the growth of Polyplastron multivesiculatum previously grown without protozoa on Hungatetype salts medium. The experiment was made in caudatum-type salts medium under $95 \% \mathrm{~N}_{2}+$ $5 \% \mathrm{CO}_{2}$ inoculated with $10 \%$ of each of the protozoal cultures. - - Number of epidinia in the presence of polyplastrons.

time the number of $P$. multivesiculatum increased from 3 to $8 \mathrm{I} / \mathrm{ml}$ in 3 days with an average mean generation time of 0.6 day. In the absence of epidinia the number declined to one protozoon in $10 \mathrm{ml}$ in the same time. The epidinia were apparently engulfed, as once the $P$. multivesiculatum began to grow (day 4 ) the epidinia disappeared in 2 days, although their number remained unchanged in the absence of polyplastrons. These results show that $P$. multivesiculatum grown in the absence of epidinia still required epidinia when transferred to caudatum-type salts medium.

Similar results were obtained when the Epidinium ecaudatum caudatum (sheep form) was replaced by $E$. ecaudatum caudatum (cattle form) or E. ecaudatum tricaudatum and, in one experiment, during the fifth day an average of $2.04,0.69$ and 2.97 individuals respectively of these species of epidinia were engulfed by each polyplastron. However, the actual results obtained varied markedly between experiments and there is thought to be no essential difference in the rate of engulfment of the three epidinia. The addition of Eudiplodinium maggii only caused a slight ( 50 to $100 \%$ ) increase in the number of polyplastrons during the 7 days of the experiment; Diploplastron affine had no stimulatory effect.

\section{DISCUSSION}

The results quoted above provide detailed confirmation of those of Eadie (1962, 1967) in which she showed that in the rumen the protozoa of a type A population (i.e. Polyplastron multivesiculatum, Diploplastron affine and Ophryoscolex tricoronatus) were dominant over those of a type B population (i.e. Eudiplodinium maggii, Epidinium spp., Eremoplastron spp. and Ostracodinium spp.) and that this was caused by predation of $P$. multivesiculatum on the type B protozoa. However, the present results also show that, under certain conditions, $P$. multivesiculatum has an absolute requirement for growth for at least one type B protozoon/day. The time taken for $P$. multivesiculatum grown in vitro in the absence of other protozoa to become predatory upon epidinia was longer than that found in vivo (i.e. 3 to 5 days compared with $\mathrm{I}$ to 2 days), but this lag may have been due in part to placing the 
polyplastrons in fresh medium: there was a 2-day lag before growth began in the experiment shown in Fig. 6, where the polyplastrons were grown in the presence of epidinia throughout. Although conditions are very different in vivo and in vitro, this result suggests that $P$. multivesiculatum growing in vivo may be better able to engulf type B protozoa even though it has had no recent contact with them. This is supported by the observation that $P$. multivesiculatum growing in vivo in a stable type A population is intermediate in size between this protozoon growing in the presence or absence of epidinia in vitro (i.e. $175 \mu \mathrm{m}$ long compared with 205 or $\mathrm{I} 23 \mu \mathrm{m})$. The specificity of the requirement for epidinia compared with entodinia remains unexplained, although it may be related to the observation that the epidinia, but not the entodinia, have many bacteria attached to their surface (G. S. Coleman \& F. J. Hall, unpublished observation). It is possible that $P$. multivesiculatum recognizes an epidinium as a bacterium and engulfs it along with the bacteria it takes up as a source of protein, etc.

The finding that the protozoa which support growth of another come from one or two genera only is fairly common in studies with carnivorous ciliates. Claff, Dewey \& Kidder (194I) showed that Bresslaua vorax and B. insidiatrix required Glaucoma pyriformis and could not use Tetrahymena, and Dewey \& Kidder (1940) found that Perispira ovum only utilized euglenoid flagellates. Similarly, Didinium nasutum has an absolute requirement for Paramecium and cannot survive in its absence (Kudo, I946). This specificity for other protozoa by carnivorous protozoa contrasts with the lack of specificity in the engulfment of bacteria shown by the rumen ciliates Entodinium caudatum and E. simplex (Coleman, 1964, I972) and by Colpidium colpoda (Burbank, 1942) which would grow in the presence of many different bacterial species, although not all were equally effective.

We wish to thank Dr P. Kemp for inserting permanent rumen cannulae into the sheep used to provide rumen fluid for protozoal cultures, Mr A. L. Gallup for taking the photomicrographs, Dr J. M. Eadie for identifying some of the protozoal species, Miss S. A. Holdgate for providing cultures of diplodinia, and the members of the Sub-Department of Chemical Microbiology, Biochemistry Department, University of Cambridge, for their helpful advice and criticism.

\section{REFERENCES}

Burbank, W. D. (I942). Physiology of the ciliate Colpidium colpoda. I. The effect of various bacteria as food on the division rate of Colpidium colpoda. Physiological Zoology 15, 342-362.

Claff, C. L., Dewey, V. C. \& Kidder, G. W. (I94I). Feeding mechanisms and nutrition in three species of Bresslaua. Biological Bulletin, Marine Biological Laboratory, Woods Hole, Massachusetts 81, 22 I-234.

Clarke, R. T. J. (1963). The cultivation of some rumen oligotrich prototozoa. Journal of General Microbiology 33, 40I-408.

Coleman, G. S. (1958). Maintenance of oligotrich protozoa from the sheep rumen in vitro. Nature, London I82, I I O4-I 105 .

Coleman, G.S. (1960). The cultivation of sheep rumen oligotrich protozoa in vitro. Journal of General Microbiology 22, 555-563.

Coleman, G.S. (1964). The metabolism of Escherichia coli and other bacteria by Entodinium caudatum. Journal of General Microbiology 37, 209-223.

Coleman, G. S. (1969). The cultivation of the rumen ciliate Entodinium simplex. Journal of General Microbiology $57,8 \mathrm{I}-90$.

Coleman, G. S. (1970). The cultivation of the rumen ciliate Polyplastron multivesiculatum. Journal of General Microbiology 6r, iv.

Coleman, G. S. (197I). The cultivation of rumen entodiniomorphid protozoa. In Isolation of Anaerobes, pp. I 59-I76. Edited by D. A. Shapton and R. B. Board. London and New York: Academic Press.

Coleman, G. S. (1972). The metabolism of starch, glucose, amino acids, purines, pyrimidines and bacteria by the rumen ciliate Entodinium simplex. Journal of General Microbiology, 71, I I7-I 3 I. 
Crawley, H. (1923). Evolution in the ciliate family Ophryoscolecidae. Proceedings of Academy of Natural Sciences of Philadelphia 75, 393-412.

DeWEy, V. C. \& KidDER, G. W. (1940). Growth studies on ciliates. 6. Diagnosis, sterilization and growth characteristics of Perispira ovum. Biological Bulletin, Marine Biological Laboratory, Woods Hole, Massachusetts 79. 255-271.

Dogiel, V. A. (1927). Monographie der Familie Ophryoscolecidae. Archiv für Protistenkunde 59, I-227.

EADIE, J. M. (1962). Inter-relationships between certain rumen ciliate protozoa. Journal of General Microbiology 29, 579-588.

EADIE, J. M. (1967). Studies on the ecology of certain rumen ciliate protozoa. Journal of General Microbiology 49, $175-194$.

Gutierrez, J. \& Davis, R. E. (1962). Culture and metabolism of the rumen ciliate Epidinium ecaudatum Crawley. Applied Microbiology 10, 305-308.

HunGate, R. E. (1942). The culture of Eudiplodinium neglectum, with experiments on the digestion of cellulose. Biological Bulletin, Marine Biological Laboratory, Woods Hole, Massachusetts, 83, 303-319.

Hungate, R. E. (1943). Further experiments on the cellulose digestion by the protozoa in the rumen of cattle. Biological Bulletin, Marine Biological Laboratory, Woods Hole, Massachusetts, 84, I57-163.

Hungate, R. E. (1955). Mutualistic intestinal protozoa. In Biochemistry and Physiology of Protozoa, vol. 2, pp. I 59-199. Edited by S. H. Hutner and A. Lwoff. New York and London: Academic Press.

JaRvis, B. D. W. \& Hungate, R. E. (1968). Factors influencing agnotiobiotic cultures of the rumen ciliate Entodinium simplex. Applied Microbiology 16, 1044-1052.

Kofoid, C. A. \& MacLennan, R. F. (1933). Ciliates from Bos indicus Linn. 3 Epidinium Crawley, Epiplastron Gen. Nov., and Ophryoscolex Stein. University of California Publications in Zoology 39, $1-33$.

KUdo, R. R. (1946). Protozoology. Illinois, U.S.A.: C. C. Thomas.

MAH, R. A. (1964). Factors influencing the in vitro culture of the rumen ciliate Ophryoscolex purkynei Stein. Journal of Protozoology II, 546-552.

SHARP, R. G. (1914). Diplodinium ecaudatum with an account of its neuromotor apparatus. University of California Publications in Zoology 13, 43-122.

TOMPKIN, R. B., PURSER, D. B. \& WeISER, H. H. (I966). Influence of rumen fluid source upon establishment and cultivation in vitro of the rumen protozoon Entodinium. Journal of Protozoology 13, 55-58. 